\title{
MOA-2016-BLG-319Lb: Microlensing Planet Subject to Rare Minor-image Perturbation Degeneracy in Determining Planet Parameters
}

\author{
Cheongho $\operatorname{Han}^{1}$ (D), Ian A. Bond ${ }^{2,24}$, Andrew Gould ${ }^{3,4,5,25}$, \\ and
}

Michael D. Albrow ${ }^{6}$ (D), Sun-Ju Chung ${ }^{3,7}$ (D), Youn Kil Jung ${ }^{3}$, Kyu-Ha Hwang ${ }^{3}$ (D), Chung-Uk Lee ${ }^{3}$, Yoon-Hyun Ryu ${ }^{3}$ (D), In-Gu Shin ${ }^{8}$ (D), Yossi Shvartzvald ${ }^{9}$ (D), Jennifer C. Yee ${ }^{8}$ (D), Sang-Mok Cha ${ }^{3,10}$, Dong-Jin Kim ${ }^{3}$, Hyoun-Woo Kim ${ }^{3}$, Seung-Lee Kim ${ }^{3,7}$, Dong-Joo Lee ${ }^{3}$, Yongseok Lee ${ }^{3,10}$, Byeong-Gon Park ${ }^{3,7}$, Richard W. Pogge ${ }^{4}$ (D), Chun-Hwey Kim ${ }^{11}$ (iD (The KMTNet Collaboration),

and

Fumio Abe ${ }^{12}$, Richard Barry ${ }^{13}$, David P. Bennett ${ }^{13,14}$ (D), Aparna Bhattacharya ${ }^{13,14}$, Martin Donachie $^{15}$, Akihiko Fukui ${ }^{16}$ (D), Yuki Hirao $^{17}$, Yoshitaka Itow ${ }^{12}$, Kohei Kawasaki ${ }^{17}$, Iona Kondo ${ }^{17}$, Naoki Koshimoto ${ }^{18,19}$ (i), Man Cheung Alex Li ${ }^{15}$, Yutaka Matsubara $^{12}$, Yasushi Muraki ${ }^{12}$, Shota Miyazaki ${ }^{17}$ (D), Masayuki Nagakane ${ }^{17}$, Clément Ranc ${ }^{13}$, Nicholas J. Rattenbury ${ }^{15}$ (D), Haruno Suematsu $^{17}$, Denis J. Sullivan ${ }^{20}$, Takahiro Sumi ${ }^{17}$, Daisuke Suzuki ${ }^{21}$ (D) Paul J. Tristram ${ }^{22}$, and Atsunori Yonehara ${ }^{23}$ (The MOA Collaboration)

\author{
${ }^{1}$ Department of Physics, Chungbuk National University, Cheongju 28644, Republic of Korea; cheongho@astroph.chungbuk.ac.kr \\ ${ }^{2}$ Institute of Natural and Mathematical Sciences, Massey University, Auckland 0745, New Zealand \\ ${ }^{3}$ Korea Astronomy and Space Science Institute, Daejon 34055, Republic of Korea \\ ${ }^{4}$ Department of Astronomy, Ohio State University, 140 W. 18th Ave., Columbus, OH 43210, USA \\ ${ }^{5}$ Max Planck Institute for Astronomy, Königstuhl 17, D-69117 Heidelberg, Germany \\ ${ }^{6}$ University of Canterbury, Department of Physics and Astronomy, Private Bag 4800, Christchurch 8020, New Zealand \\ ${ }^{7}$ Korea University of Science and Technology, 217 Gajeong-ro, Yuseong-gu, Daejeon, 34113, Republic of Korea \\ ${ }^{8}$ Harvard-Smithsonian Center for Astrophysics, 60 Garden St., Cambridge, MA 02138, USA \\ ${ }^{9}$ IPAC, Mail Code 100-22, Caltech, 1200 E. California Blvd., Pasadena, CA 91125, USA \\ ${ }^{10}$ School of Space Research, Kyung Hee University, Yongin, Kyeonggi 17104, Republic of Korea \\ ${ }^{11}$ Department of Astronomy \& Space Science, Chungbuk National University, Cheongju 28644, Republic of Korea \\ ${ }^{12}$ Institute for Space-Earth Environmental Research, Nagoya University, Nagoya 464-8601, Japan \\ ${ }^{13}$ Code 667, NASA Goddard Space Flight Center, Greenbelt, MD 20771, USA \\ ${ }^{14}$ Department of Astronomy, University of Maryland, College Park, MD 20742, USA \\ 15 Department of Physics, University of Auckland, Private Bag 92019, Auckland, New Zealand \\ ${ }^{16}$ Okayama Astrophysical Observatory, National Astronomical Observatory of Japan, 3037-5 Honjo, Kamogata, Asakuchi, Okayama 719-0232, Japan \\ ${ }^{17}$ Department of Earth and Space Science, Graduate School of Science, Osaka University, Toyonaka, Osaka 560-0043, Japan \\ ${ }^{18}$ Department of Astronomy, Graduate School of Science, The University of Tokyo, 7-3-1 Hongo, Bunkyo-ku, Tokyo 113-0033, Japan \\ ${ }^{19}$ National Astronomical Observatory of Japan, 2-21-1 Osawa, Mitaka, Tokyo 181-8588, Japan \\ ${ }^{20}$ School of Chemical and Physical Sciences, Victoria University, Wellington, New Zealand \\ ${ }^{21}$ Institute of Space and Astronautical Science, Japan Aerospace Exploration Agency, 3-1-1 Yoshinodai, Chuo, Sagamihara, Kanagawa, 252-5210, Japan \\ ${ }_{22}^{2}$ University of Canterbury Mt. John Observatory, P.O. Box 56, Lake Tekapo 8770, New Zealand \\ ${ }^{23}$ Department of Physics, Faculty of Science, Kyoto Sangyo University, 603-8555 Kyoto, Japan \\ Received 2018 August 19; revised 2018 September 17; accepted 2018 September 20; published 2018 October 26
}

\begin{abstract}
We present the analysis of the planetary microlensing event MOA-2016-BLG-319. The event light curve is characterized by a brief ( $\sim 3$ days) anomaly near the peak produced by minor-image perturbations. From modeling, we find two distinct solutions that describe the observed light curve almost equally as well. From the investigation of the lens-system configurations, we find that the confusion in the lensing solution is caused by the degeneracy between the two solutions resulting from the source passages on different sides of the planetary caustic. These degeneracies can be severe for major-image perturbations, but it is known that they are considerably less severe for minor-image perturbations. From the comparison of the lens-system configuration with those of two previously discovered planetary events, for which similar degeneracies were reported, we find that the degeneracies are caused by the special source trajectories that passed the star-planet axes at approximately right angles. By conducting a Bayesian analysis, it is estimated that the lens is a planetary system in which a giant planet with a mass $M_{\mathrm{p}}=0.62_{-0.33}^{+1.16} M_{\mathrm{J}}\left(0.65_{-0.35}^{+1.21} M_{\mathrm{J}}\right)$ is orbiting a low-mass M-dwarf host with a mass $M_{\mathrm{h}}=0.15_{-0.08}^{+0.28} M_{\odot}$. Here the planet masses in and out of the parentheses represent the masses for the individual degenerate solutions. The projected host-planet separations are $a_{\perp} \sim 0.95$ and $\sim 1.05 \mathrm{au}$ for the two solutions. The identified degeneracy indicates the need to check similar degeneracies in future analyses of planetary lensing events with minor-image perturbations.
\end{abstract}

Key words: gravitational lensing: micro - planetary systems

\footnotetext{
24 MOA Collaboration.

25 KMTNet Collaboration.
}

\section{Introduction}

Microlensing signals of planets are often described by the phrase "a brief anomaly" to the lensing light curve produced by 
the host of the planet. However, this phrase is oversimplified because the pattern of planet-induced anomalies greatly varies depending on the configurations of lens systems. Furthermore, planetary signals in some cases of lens configurations can be confused with anomalies produced by other reasons and this induces a degeneracy problem in which multiple interpretations exist for an observed anomaly pattern. Therefore, identifying the origins of degeneracies in various lens configurations is important to find correct interpretations of microlensing planets by enabling one to check similar degeneracies in future analyses.

The degeneracies in the interpretation of planetary signals are broadly classified into two categories: "intrinsic" and "accidental". The intrinsic degeneracies are caused by the symmetry of the lens equation, which describes the mapping from the source position on the lens plane into the image position on the source plane. The most well known of these is the "close/wide" degeneracy in which a pair of planetary models with projected separations from the host (normalized to the angular Einstein radius $\left.\theta_{\mathrm{E}}\right) s$ and $s^{-1}$ result in very similar anomaly patterns. This degeneracy was first found by Griest \& Safizadeh (1998) for a specific case of a planetary lens system and later extended to general binary lenses by Dominik (1999) and further investigated by An (2005).

Accidental degeneracies, on the other hand, occur due to the fortuitous alignment of lensing magnification patterns arising from unrelated lens configurations. It was pointed out by Gaudi (1998) that a subset of binary-source events, for which the flux ratio between the binary-source stars is small and the lens approaches close to the faint source companion, can produce short-term anomalies, which are similar to those of planetinduced anomalies. These planet/binary-source degeneracies were actually found for MOA-2012-BLG-486 (Hwang et al. 2013) and OGLE-2015-BLG-1459 (Hwang et al. 2018c) for which the degeneracies were difficult to be resolved just based on the lensing light curves and could be resolved with additional data acquired from multi-band observations. Han \& Gaudi (2008) pointed out another type of accidental degeneracy in which planetary signals can be imitated by those produced by binaries composed of roughly equal masses. Such degeneracies were demonstrated for OGLE-2011-BLG-0526, OGLE-2011BLG-0950/MOA-2011-BLG-336 (Choi et al. 2012), and OGLE-2015-BLG-1212 (Bozza et al. 2016). In addition, incomplete coverage of the planet-induced anomalies can cause degeneracies in interpreting anomalies as demonstrated in the case of OGLE-2012-BLG-0455/MOA-2012-BLG-206 (Park et al. 2014; Hwang et al. 2018a).

Gaudi \& Gould (1997, hereafter GG1997) predicted another type of accidental degeneracy, in which two planetary lens configurations had similar $(s, q, \alpha)$ but with the source passing on different sides of the caustic. Here $q$ is the planet/host mass ratio and $\alpha$ represents the angle between the trajectory of the source and the axis connecting the planet and its host (source trajectory angle). Gould \& Loeb (1992) argued that (under the assumption that the source passed directly over the caustic) one could read off the values of $(s, \alpha)$ from the three Paczyński (1986) parameters of the point-lens fit $\left(t_{0}, u_{0}, t_{\mathrm{E}}\right)$ and the time of the planetary perturbation $\left(t_{\text {anom }}\right)$ using the relations

$$
s-\frac{1}{s}=\left(u_{0}^{2}+\tau_{\text {anom }}^{2}\right)^{1 / 2} ; \quad \tau_{\text {anom }}=\frac{t_{\text {anom }}-t_{0}}{t_{\mathrm{E}}},
$$

and

$$
\tan \alpha=\frac{u_{0}}{\tau_{\text {anom }}}
$$

If the caustic is relatively small, this approach is approximately accurate, even if the source only passes near the caustic. However, GG1997 recognized that this would lead to two slightly different solutions depending on whether the source passes on one side of the caustic or the other. They pointed out that the degeneracy would be severe for perturbations produced by planets with projected planet-host separations greater than the angular Einstein radius ( $s>1$, "wide" planet): "major image perturbations". For "minor-image perturbations", which are produced by "close" planets with $s<1$, on the other hand, it was thought that the degeneracy would be considerable less severe. This is mainly because of the qualitative difference in the caustic structures between the wide and close planetary systems, in which a wide planet induces a single set of planetary caustics, and a close planet induces two sets. In the Appendix, we review basic facts about the types of planetary anomalies caused by major- and minor-image perturbations for readers who are not familiar with microlensing jargon. Experts needn't read the Appendix section. For the major-image caustic, the magnification pattern on the near and far sides of the caustic are similar, and thus the anomalies produced by the source passing both sides of the caustic are similar to each other. For minor-image perturbations, on the other hand, the source trajectory passing the inner cusp will, in general, approach close to one of the two planetary caustics, while the source trajectory passing the outer cusp will approach the other caustic. As a result, the degeneracy would be generally resolvable from the presence (absence) and/or timing of the anomalies produced by the individual caustics.

Degeneracies involved with planetary caustics have been demonstrated for actual lensing events and new types of degeneracies are additionally found with the increasing number of microlensing planets. An example of the GG1997 degeneracy was recently found for the planetary event OGLE-2017BLG-0173 (Hwang et al. 2018d), for which there existed three degenerate solutions, and among them two solutions were caused by the degeneracy predicted by GG1997. We note that the other solution results from a new discrete degeneracy between the solution in which the caustic is fully enveloped ("Cannae" solution) and the solution in which only one side of the caustic is enveloped ("von Schlieffen" solution): "Hollywood" degeneracy. The two solutions resulting from the Hollywood degeneracy have different mass ratios because the source passes through the caustic in different places. Skowron et al. (2018) found a more specific case of GG1997 degeneracies from the analysis of the planetary lensing event OGLE-2017-BLG-0373. This so-called "caustic-chiral" degeneracy arises when the source passes over the caustic (contrary to the GG1997 degeneracy), but there are gaps in data. In this case, the solutions have very similar $(s, \alpha)$ but substantially different $q$. Another example of the caustic-chiral degeneracy was found by Hwang et al. (2018a) for KMT-2016-BLG-0212. In addition, there is a case of the source passing through a major-image caustic and being degenerate with passing through 


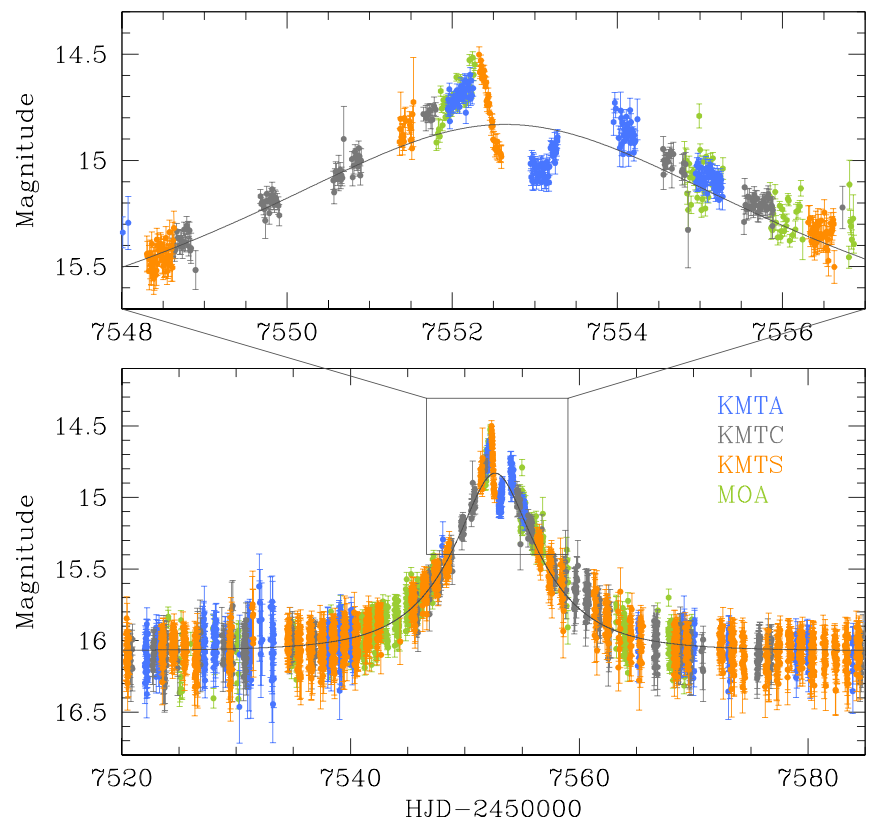

Figure 1. Light curve of the lensing event MOA-2016-BLG-319. The zoom of the anomaly near the peak is shown in the upper panel. Superposed on the data points represents the point-source point-lens model.

a minor-image caustic. This degeneracy was identified for KMT-2016-BLG-1107 by Hwang et al. (2018b).

In this work, we analyze the planetary event MOA-2016BLG-319. The light curve is characterized by a short-term anomaly near the peak produced by the minor-image perturbation. Contrary to the expectation that interpreting minor-image perturbations would not suffer from degeneracies, we find two discrete solutions that describe the observed light curve almost equally well. Similar degeneracies in minor-image perturbations were reported for two planetary events OGLE-2016-BLG-1067 (Calchi Novati et al. 2018) and OGLE-2012-BLG-0950 (Koshimoto et al. 2017). By analyzing the similarity between the anomalies of the events, we investigate the origin of the degeneracy.

\section{Observations}

We identify the case of degeneracies in minor-image perturbations from the analysis of the lensing event MOA2016-BLG-319. Figure 1 shows the event light curve. The source star of the event is located at the Galactic bulge field with equatorial coordinates (R.A., decl. $)_{\mathrm{J} 2000}=(17: 54: 58.13$, $-29: 45: 01.67)$. The corresponding galactic coordinates are $(l, b)=\left(0.35,-2^{\circ} .17\right)$. The magnification of the source star induced by lensing was first detected and announced to the microlensing community on 2016 June 13, HJD $^{\prime}=$ HJD $-2,450,000 \sim 7552$, by the Microlensing Observations in Astrophysics (MOA) survey (Bond et al. 2001; Sumi et al. 2003). The MOA survey used the $1.8 \mathrm{~m}$ telescope located at Mt. John Observatory, New Zealand. The day of the event alert approximately corresponds to not only the time of the light curve peak but also the start of a short-term anomaly, which lasted for $\sim 3$ days during $7551.5 \lesssim \mathrm{HJD}^{\prime} \lesssim 7554.5$. However, it was difficult to notice the anomaly because the MOA survey could not cover the event for four consecutive nights before the anomaly, and the photometry of data during the anomaly was
Table 1

Data Sets Used in the Analysis

\begin{tabular}{lcr}
\hline \hline Data Set & Range & $N_{\text {data }}$ \\
\hline MOA & $7502 \lesssim \mathrm{HJD}^{\prime} \lesssim 7569$ & 757 \\
KMTA (BLG02) & $7440 \lesssim \mathrm{HJD}^{\prime} \lesssim 7676$ & 798 \\
KMTA (BLG42) & $7443 \lesssim \mathrm{HJD}^{\prime} \lesssim 7676$ & 701 \\
KMTC (BLG02) & $7439 \lesssim \mathrm{HJD}^{\prime} \lesssim 7675$ & 1143 \\
KMTC (BLG42) & $7439 \lesssim \mathrm{HJD}^{\prime} \lesssim 7675$ & 1006 \\
KMTS (BLG02) & $7441 \lesssim \mathrm{HJD}^{\prime} \lesssim 7681$ & 1394 \\
KMTS (BLG42) & $7441 \lesssim \mathrm{HJD}^{\prime} \lesssim 7681$ & 1472 \\
\hline
\end{tabular}

Note. $\mathrm{HJD}^{\prime}=\mathrm{HJD}-2,450,000$.

not good enough to delineate the anomaly pattern. As a result, little attention was paid to the event during the progress of the event.

The scientific importance of the event was noticed with the additional data acquired by Korea Microlensing Telescope Network (KMTNet) survey (Kim et al. 2016) conducted using three $1.6 \mathrm{~m}$ telescopes. The individual KMTNet telescopes are positioned at the Cerro Tololo Interamerican Observatory, Chile (KMTC), the South African Astronomical Observatory, South Africa (KMTS), and the Siding Spring Observatory, Australia (KMTA). The alert system of the KMTNet survey started from the 2018 season (Kim et al. 2018b) and the progress of the event was not known in real time at the time of the event during the 2016 season. From the analysis of lensing events identified by applying the Event Finder algorithm (Kim et al. 2018a, 2018b) to the 2016 season data, it was found that KMTNet data densely covered the light curve peak which clearly showed a short-term anomaly. See the zoom of the light curve around the anomaly region presented in the upper panel of Figure 1. The event was identified by KMTNet as KMT-2016-BLG-1816.

In Table 1, we present the data sets used in the analysis. MOA observations of the event were conducted in a customized $R$ band with a cadence of $1 \mathrm{hr}$. KMTNet observations were conducted mostly in $I$ band with occasional observations in $V$ band to measure the source color. The event was in the KMTNet BLG02 and BLG42 fields, which were monitored with a cadence of $0.5 \mathrm{hr}$ by the individual telescopes. For the period from April $23\left(\mathrm{HJD}^{\prime} \sim 2457501\right)$ to June 16 $\left(\mathrm{HJD}^{\prime} \sim 2457555\right)$, the cadence of KMTS and KMTA was increased in order to support Kepler K2 C9 campaign (Gould \& Horne 2013). While the event does not lie in the K2 field, the anomaly coverage serendipitously benefited from this cadence increase. The columns "range" and " $N_{\text {data }}$ " indicate the time range of the data sets used for analysis and the number of data points constituting the individual data sets, respectively. We set the range of the MOA data in the region around event because the baseline data exhibit considerable fluctuation.

Photometry of data are processed using the codes of the individual groups: Bond et al. (2001) for the MOA survey and Albrow et al. (2009) for the KMTNet survey. Both codes utilize the difference imaging method developed by (Alard \& Lupton 1998). Errorbars are normalized using the recipe explained in Yee et al. (2012).

\section{Light Curve Analysis}

The light curve shows a pronounced dip. Such a dip feature in lensing light curves can only be produced by minor image perturbations. There are two possibilities in the lens-system 

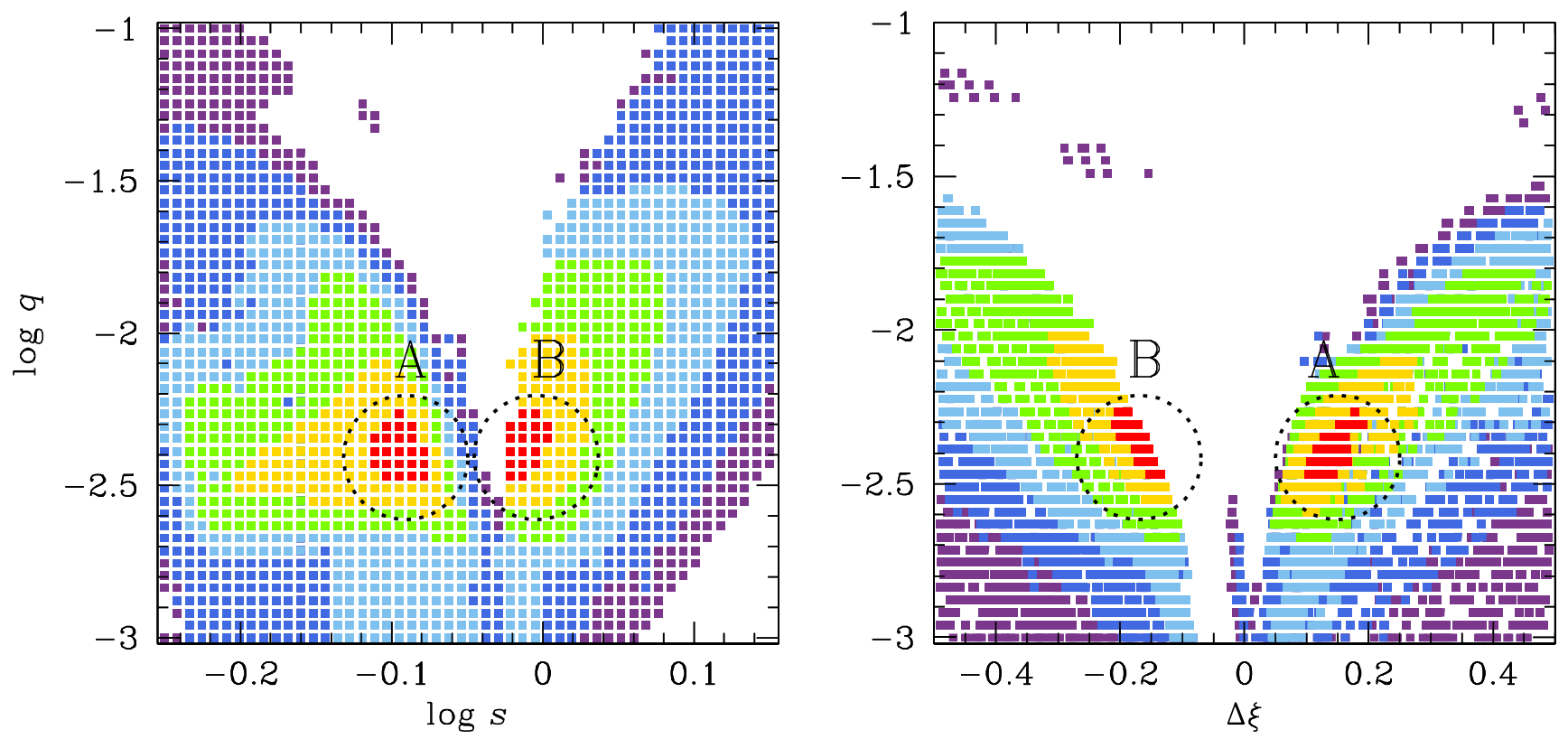

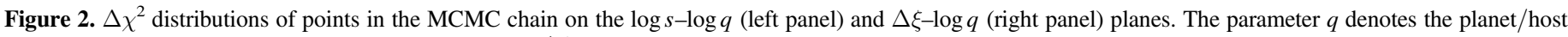

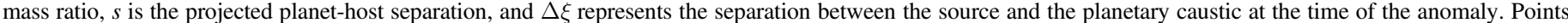

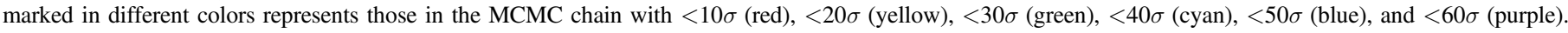

configuration. First, the minor image gives rise to two triangular planetary caustics with a magnification dip between them. Second, there is a six-sided resonant caustic whose "back end" consists of two caustic wings separated by a dip. See Figure 4 of Gaudi (2012) for the variation of planetary microlens caustics.

Although some lens configurations are excluded in advance based on the previously well-studied origins of degeneracies, interpreting the anomaly may be subject to unknown types of degeneracies. We, therefore, conduct a thorough grid search for the planetary lensing parameters $s$ and $q$. Besides these planetary parameters, one needs additional lensing parameters to model the observed light curve. These parameters describe the source star's approach to the lens including the time of the closest approach, $t_{0}$, the lens-source separation at that time, $u_{0}$ (impact parameter), the event timescale, $t_{\mathrm{E}}$ (Einstein timescale), and the source trajectory angle. The anomaly might be produced by the crossings of the source over caustics. There is no obvious signature of caustic crossings, which usually produce sharp spike features. However, caustic-crossing features can be smooth if the source is substantially bigger than the caustic. Even if a source is smaller than an overall caustic, it could be big compared to the caustic figure that it is passing over, e.g., OGLE 2016-BLG-1195 (Bond et al. 2017) and OGLE-2016-BLG-1195 (Shvartzvald et al. 2017). These finite-source effects were theoretically predicted by Bennett \& Rhie (1996) and observationally confirmed by Beaulieu et al. (2006) for the planetary lensing event OGLE-2005-BLG-390. To account for possible finite-source effects, we include an additional parameter of the normalized source radius $\rho$, which denotes the ratio of the angular source radius $\theta_{*}$ to the angular Einstein radius $\theta_{\mathrm{E}}$, i.e., $\rho=\theta_{*} / \theta_{\mathrm{E}}$. For a given set of the planetary parameters $s$ and $q$, we search for the other parameters using the Markov Chain Monte Carlo (MCMC) method. We set the ranges of the grid parameters, i.e., $s$ and $q$, wide enough to check the possibility that the anomaly is produced by binaries that have similar mass components.
In the left panel of Figure 2, we present the $\Delta \chi^{2}$ distribution of points in the MCMC chain on the $\log s-\log q$ plane acquired from the preliminary grid search. From the distribution, one first finds that the lens responsible for the anomaly is composed of two masses with a very low mass ratio of $q \sim 4 \times 10^{-3}$, suggesting that the lower-mass component of the lens is a planet. One also finds that there exist two distinct solutions centered at $(\log s, \log q) \sim(-0.09,-2.4)$ (marked by "A") and $\sim(-0.02,-2.4)$ ("B"). From further refinement of the individual local solutions by letting all parameters vary, it is found that the $\chi^{2}$ difference between the two solutions is merely $\Delta \chi^{2} \sim 1.9$. This indicates that both solutions describe the observed anomaly almost equally well, although the solution " $A$ " is slightly preferred over the solution " $\mathrm{B}$ ".

To be noted is that the degeneracy between the two solutions is different from the previously known "close/wide" degeneracy. The two solutions resulting from the close/wide degeneracy have planet-host separations $s$ and $s^{-1}$, and thus one solution has a separation smaller than $\theta_{\mathrm{E}}$, i.e., $s<1$, and the other solution has a separation greater than $\theta_{\mathrm{E}}, s>1$. In the case of MOA-2016-BLG-319, both degenerate solutions have separations $s<1$ ( $s \sim 0.82$ for the solution "A" and $s \sim 0.95$ for the solution "B") indicating that the origin of the degeneracy does not stem from the symmetry of the lens equation. $^{26}$

In Table 2, we present the best-fit lensing parameters for the two degenerate solutions. We also present the $\chi^{2}$ values of the fit for the solutions. The uncertainties of the parameters correspond to the scatter of points in the MCMC chain. We note that the lensing parameters of the two degenerate solutions are very similar to each other except for the binary separation $s$. To be also noted is that the timescale of the event, $t_{\mathrm{E}} \sim 8.6$

\footnotetext{
${ }^{26}$ We note that the red zone in the $\Delta \chi^{2}$ distribution (presented in the left panel of Figure 2) covers $s=1$ and even slightly greater. If $s \sim 1$, then the lens system forms a resonant caustic, in which the central and planetary caustic merge together. In this case, the back-end of the resonant caustic still induce a dip in the light curve, even if the binary separation $s$ is greater than unity.
} 
Table 2

Best-fit Lensing Parameters

\begin{tabular}{lcc}
\hline \hline Parameter & Inner Solution (“A”) & Outer Solution (“B”) \\
\hline$\chi^{2}$ & 7308.1 & 7310.0 \\
$t_{0}\left(\mathrm{HJD}^{\prime}\right)$ & $7552.737 \pm 0.013$ & $7552.742 \pm 0.012$ \\
$u_{0}$ & $0.267 \pm 0.012$ & $0.260 \pm 0.012$ \\
$t_{\mathrm{E}}($ days $)$ & $8.60 \pm 0.26$ & $8.69 \pm 0.26$ \\
$s$ & $0.817 \pm 0.004$ & $0.945 \pm 0.008$ \\
$q\left(10^{-3}\right)$ & $3.93 \pm 0.11$ & $4.10 \pm 0.12$ \\
$\alpha(\mathrm{rad})$ & $4.646 \pm 0.006$ & $4.645 \pm 0.006$ \\
$F_{\mathrm{s}, \text { KMTC }}$ & $6.25 \pm 0.08$ & $6.23 \pm 0.08$ \\
$F_{\mathrm{b}, \text { KMTC }}$ & $-0.10 \pm 0.11$ & $-0.10 \pm 0.11$ \\
\hline
\end{tabular}

Note. $\mathrm{HJD}^{\prime}=\mathrm{HJD}-2,450,000$.

days, is short. As a result, higher-order effects induced by the orbital motion of the Earth, microlens-parallax effect (Gould 1992), or that of the lens, lens-orbital effect (Dominik 1998), is not important in describing the observed light curve. Also presented in the table are the flux values of the source, $F_{\mathrm{s}, \text { KMTC}}$, and blend, $F_{\mathrm{b}, \text { KMTC }}$, as measured from the pyDIA photometry of the KMTC data set. We note that the blend flux has a slightly negative value but it is consistent to be zero within the measurement error. These measured values of $F_{\mathrm{s}, \mathrm{KMTC}}$ and $F_{\mathrm{b}, \mathrm{KMTC}}$ indicate that the flux from the source dominates the blended flux.

In Figure 3, we present model light curves for both solutions plotted over the observed data points. Except for the very short period around $\mathrm{HJD}^{\prime} \sim 7553.4$, the two model light curves are so similar to each other that it is difficult to distinguish them within the line width, indicating that the degeneracy between the two solutions is very severe. This can be also seen in the lower two panels in which the residuals from the individual solutions are presented.

Figure 4 shows the configurations of the lens system for the individual solutions. In each panel, the line with an arrow represents the source trajectory, the small dots marked by $M_{1}$ and $M_{2}$ indicate the positions of the lens components, and the closed figures composed of concave curves represent the caustic. The upper and lower panels are the configurations for the solutions "A" and "B", respectively. We draw contours of magnification to show the region of anomaly around the caustics. For the solution "A", the caustic is composed of three sets in which the small caustic located close to the host is the central caustic, and the two caustics located away from the host are the planetary caustics. According to this solution, the anomaly was produced by the passage of the source through the region between the central and planetary caustics. The negative deviation of the anomaly was produced during the time when the source passed the negative perturbation region between the central and planetary caustics. Because the source trajectory passed the inner region of the planetary caustic with respect to the planet host, we refer to this solution as "inner solution". For solution "B", on the other hand, the lens system produces a single resonant caustic. This results from the merging of the central and planetary caustics because of the proximity of the planet-host separation to unity, $s \sim 0.95$. According to this solution, the source trajectory passed the back-end of the caustic and the negative deviation occurred during the time when the source passed the region extending from the caustic end. Because the source trajectory passed the outer region of

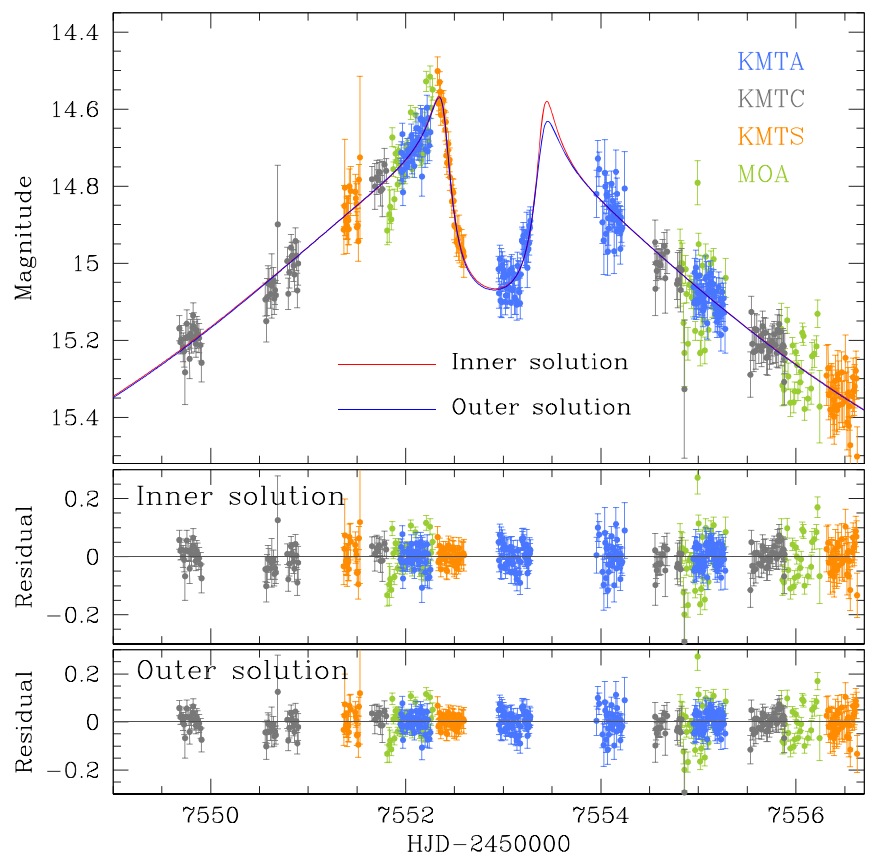

Figure 3. Two model light curve resulting from the two degenerate solutions: "inner solution" and "outer solution". The lower panels show the residuals from the individual solutions. The lens-system configurations corresponding to the individual solutions are presented in Figure 4.
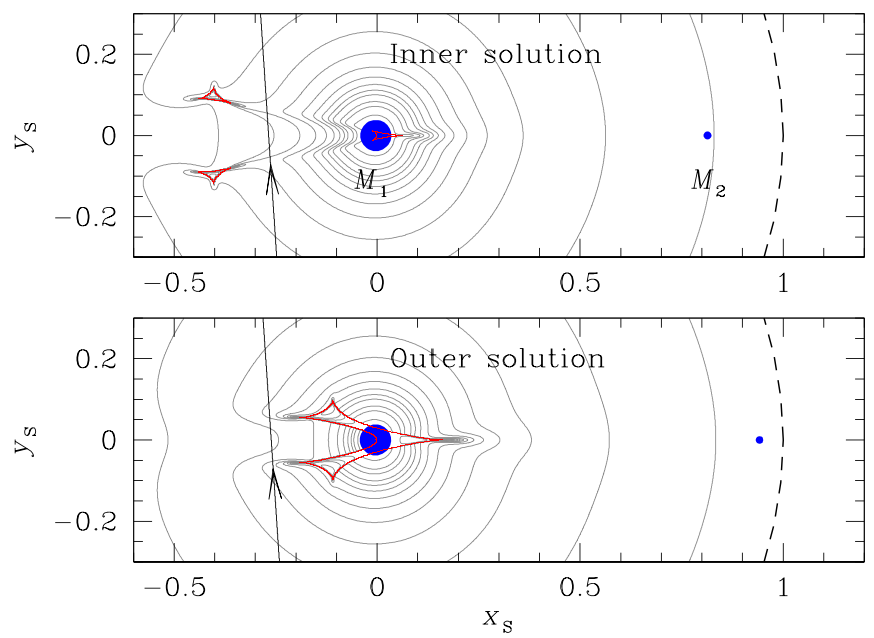

Figure 4. Lens-system configurations for the two degenerate solutions seen on the source plane. For each panel, the curve with an arrows represents the source trajectory and the red closed curves represent caustics. The two dots marked by $M_{1}$ and $M_{2}$ denote the lens components, where $M_{1}>M_{2}$. The dashed circle centered at $M_{1}$ represents the Einstein ring. Contours of lensing magnification are drawn to show the region of anomaly around caustics.

the caustic (with respect to the planet host), we refer to this solution as "outer solution".

To better understand the origin of the degeneracy between the two solutions, in Figure 5, we present the lens-system configurations seen on the lens plane. In the plot, the planet host is located at the origin and the line with an arrow represents the path of the source. The two solid curves with arrows represent the paths of the images produced by the host. The red and blue dots represent the planet positions for the inner and outer solutions, respectively. From the configurations, it is found that, for both solutions, the planet is located 


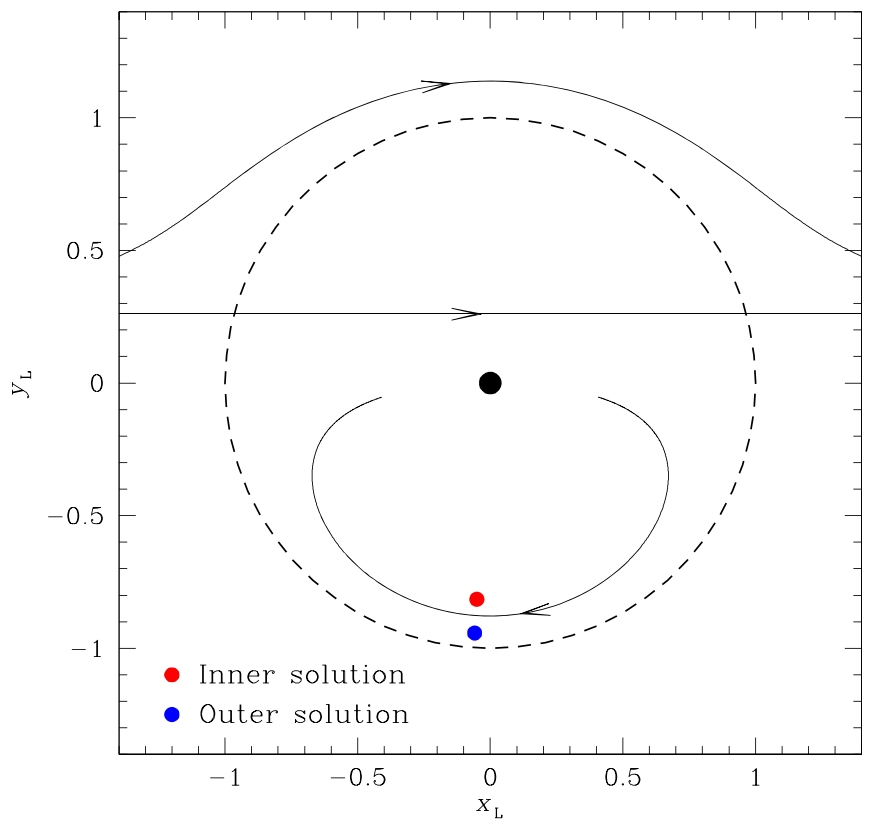

Figure 5. Lensing system configurations for the two degenerate solutions seen on the lens plane. The coordinates are centered at the position of the planet host (black dot) and the dashed circle represents the Einstein ring. The solid line with an arrow represents the path of the source. The two solid curves with arrows represent the paths of the images produced by the planet host. The red and blue dots represent the planet positions for the inner and outer solutions, respectively.

close to the minor image produced by the host at the time of the perturbation. The difference in the configurations between the two solutions is that the planet is located inside the minor image (with respect to the host) for the "inner solution", while it is located outside of the image for the "outer solution". This indicates that the similarity between the two solutions is caused by the degeneracy in minor-image perturbations. We note that the lenssystem configuration is similar to that presented in Figure 1 of GG1997 except that the positions of planets are different.

The fact that the two solutions are originated from the GG1997 degeneracy can also be seen in the right panel of Figure 2, in which we plot $\Delta \chi^{2}$ distribution of MCMC points on the $\Delta \xi-\log q$ plane. Here $\Delta \xi$ represents the separation between the source and the planetary caustic at the time of the anomaly. The separation $\Delta \xi$ is determined from the lensing parameters by

$$
\Delta \xi=\frac{u_{0}}{\sin \alpha}-\left(s-\frac{1}{s}\right),
$$

where the former term on the right side, i.e., $u_{0} / \sin \alpha$, represents the separation between the source and planet host at the time of the anomaly and the latter term, i.e., $s-1 / s$, denotes the separation between the caustic center and the host. We note that similar plots are presented in Figure 4 of Hwang et al. (2018d) and Figure 5 of Skowron et al. (2018). From the plot, it is found that the degenerate solutions have similar separations $|\Delta \xi| \sim 0.15$ but with opposite signs, indicating that the source stars of the individual solutions approach the opposite sides of the caustic with similar separations from the caustic.

Despite that degeneracies are thought to be considerably less severe for minor-image perturbations compared to major-image perturbations, similar degeneracies in minor-image perturbations were reported by Calchi Novati et al. (2018) and Koshimoto et al. (2017) for the planetary events OGLE-2016-BLG-1067 and OGLE-2012-BLG-0950, respectively. We, therefore, compare the lens-system configurations of MOA-2016-BLG-319 with the two other events in order to find the cause of the degeneracy. The lens-system configurations of OGLE-2016BLG-1067 are presented in Figures 3 and 4 of Calchi Novati et al. (2018). We note that they presented 8 degenerate configurations, among which a four-fold degeneracy is caused by the space-based parallax measurement (Refsdal 1966; Gould 1994) and the other two-fold degeneracy is relevant to the minor-image perturbation. The configurations of OGLE2012-BLG-0950 are presented in Figure 2 of Koshimoto et al. (2017).

From comparing the lens-system configurations of the events, we find one major difference and one major similarity. The difference is that the planetary caustics of the outer solution is separated from the central caustic for OGLE-2016BLG-1067, while it is merged with the central caustic for MOA-2016-BLG-319 and OGLE-2012-BLG-0950 (resonant topology). However, in the sense that the source trajectory of MOA-2016-BLG-319 and OGLE-2012-BLG-0950 passed the planetary wing of the resonant caustic, the degeneracies of the events are considered to be of the same type. The similarity is that the source stars of the events passed the planet-host axes at about right angles. The source trajectory angles are $\alpha \sim 267^{\circ}$, $\sim 278^{\circ}, \sim 292^{\circ}$ for MOA-2016-BLG-319, OGLE-2016-BLG1067, and OGLE-2012-BLG-0950, respectively. For general cases in which the entrance angle of the source is substantially different from a right angle, the source trajectory passing the inner region of the minor-image caustic produces a light curves that is distinct from a trajectory passing the outer region. On one side of the set of caustics, they will pass closer to one of the two caustics and farther from the other caustic. On the other side, the order will be inverted (for a fixed angle). This will result in different anomaly patterns, and thus one can easily distinguish the two cases if the anomaly is densely covered. In the case of a right angle source entrance, on the other hand, the source approaches the individual caustics with approximately same distances. Then, the anomaly patterns resulting from the source trajectories passing the inner and outer regions of the planetary caustic can appear to be similar. We, therefore, conclude that the degeneracies in the minor-image perturbations of both events occur because the source stars crossed the star-planet axes at approximately right angles.

\section{Source Star}

We characterize the source star based on the source flux measured in $I$ and $V$ passbands. Besides simply knowing the type of the source star, characterizing the source star is important because it may provide information about the angular Einstein radius in combination with the normalized source radius $\rho$ via the relation $\theta_{\mathrm{E}}=\theta_{*} / \rho$. For MOA-2016-BLG-319, however, the normalized source radius $\rho$ cannot be measured because deviations in the lensing light curve caused by finitesource effects cannot be firmly detected. See Figure 6, in which we plot the $\Delta \chi^{2}$ distributions of points in the MCMC chain on the $s-\rho$ parameter plane. However, the upper limit on $\rho$ can be measured and this yields a lower limit on $\theta_{\mathrm{E}}$, which may provide a constraint on the physical lens parameters. It is found that the upper limit of the normalized source radius is $\rho_{\max } \sim 0.01$ as measured at the $3 \sigma$ level. 

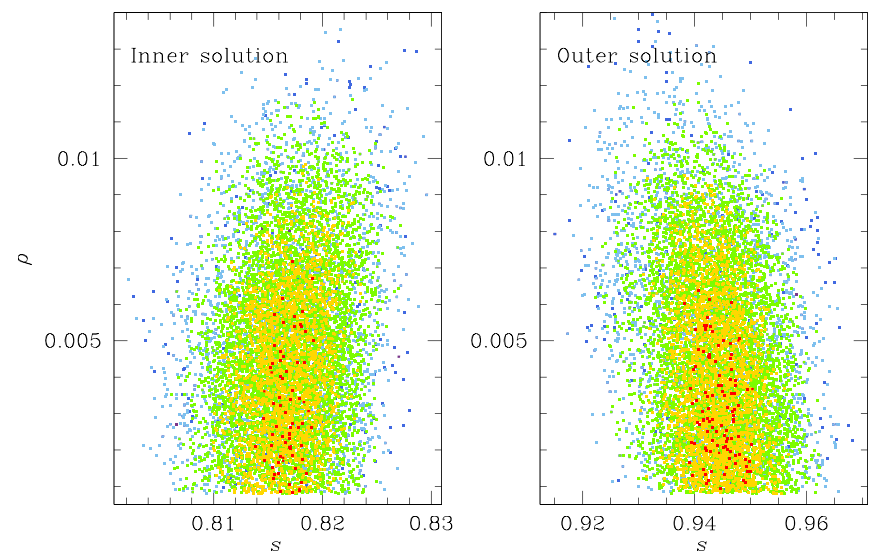

Figure 6. Distributions of $\Delta \chi^{2}$ of points in the MCMC chain on the $s-\rho$ parameter plane. Color coding represents points within $1 \sigma$ (red), $2 \sigma$ (yellow), $3 \sigma$ (green), $4 \sigma$ (cyan), and $5 \sigma$ (blue). The left and right panels are the distributions for the inner and outer solutions, respectively.

The de-reddened color $(V-I)_{0}$ and brightness $I_{0}$ of the source star are estimated using the known values of the red giant clump (RGC) centroid, $(V-I, I)_{\mathrm{RGC}, 0}=(1.06,14.41)$ (Bensby et al. 2011; Nataf et al. 2013), and the offsets in color and magnitude of the source from the RGC centroid. In Figure 7, we mark the positions of the source and RGC centroid in the color-magnitude diagram of stars located in the same field of the source. The positions of the source and the RGC centroid are $(V-I, I)=(2.00 \pm 0.04,19.01 \pm 0.01)$ and $(V-I, I)_{\mathrm{RGC}}=(2.29,15.94)$, respectively. From the color and brightness offsets, it is found that the de-reddened color and brightness of the source star are $(V-I, I)_{0}=$ $(0.77 \pm 0.04,17.49 \pm 0.01)$, respectively. The estimated dereddened color and magnitude of the source star indicate that the source is likely to be a turnoff star. We then convert the measured $V-I$ color into $V-K$ color using the $(V-I) /$ $(V-K)$ relation of Bessell \& Brett (1988). Finally, we estimate the source angular radius using the $(V-K) / \theta_{*}$ relation of Kervella et al. (2004). The estimated angular source radius is

$$
\theta_{*}=1.07 \pm 0.09 \mu \text { as. }
$$

With the measured angular source radius, the lower limit of the angular Einstein radius is set to be

$$
\theta_{\mathrm{E}, \min }=\frac{\theta_{*}}{\rho_{\max }} \sim 0.107 \text { mas. }
$$

\section{Bayesian Analysis of Lens Parameters}

In order to uniquely determine the mass, $M$, and distance to the lens, $D_{\mathrm{L}}$, it is required that both the microlens parallax $\pi_{\mathrm{E}}$ and the angular Einstein radius $\theta_{\mathrm{E}}$ are measured, from which $M$ and $D_{\mathrm{L}}$ are determined by

$$
M=\frac{\theta_{\mathrm{E}}}{\kappa \pi_{\mathrm{E}}} ; \quad D_{\mathrm{L}}=\frac{\mathrm{au}}{\pi_{\mathrm{E}} \theta_{\mathrm{E}}+\pi_{\mathrm{S}}}
$$

(Gould 2000b). Here $\kappa=4 G /\left(c^{2} \mathrm{au}\right) \sim 8.14 \operatorname{mas} M_{\odot}^{-1}$ and $\pi_{\mathrm{S}}=\mathrm{au} / D_{\mathrm{S}}$ denotes the parallax of the source located at a distance $D_{\mathrm{S}}$. For MOA-2016-BLG-319, however, neither $\pi_{\mathrm{E}}$ nor $\theta_{\mathrm{E}}$ is measured, although the upper limit of $\theta_{\mathrm{E}}$ is set. We, therefore, conduct a Bayesian analysis of Galactic lensing events to estimate $M$ and $D_{\mathrm{L}}$ based on the measured event timescale. The timescale provides a constraint on the lens

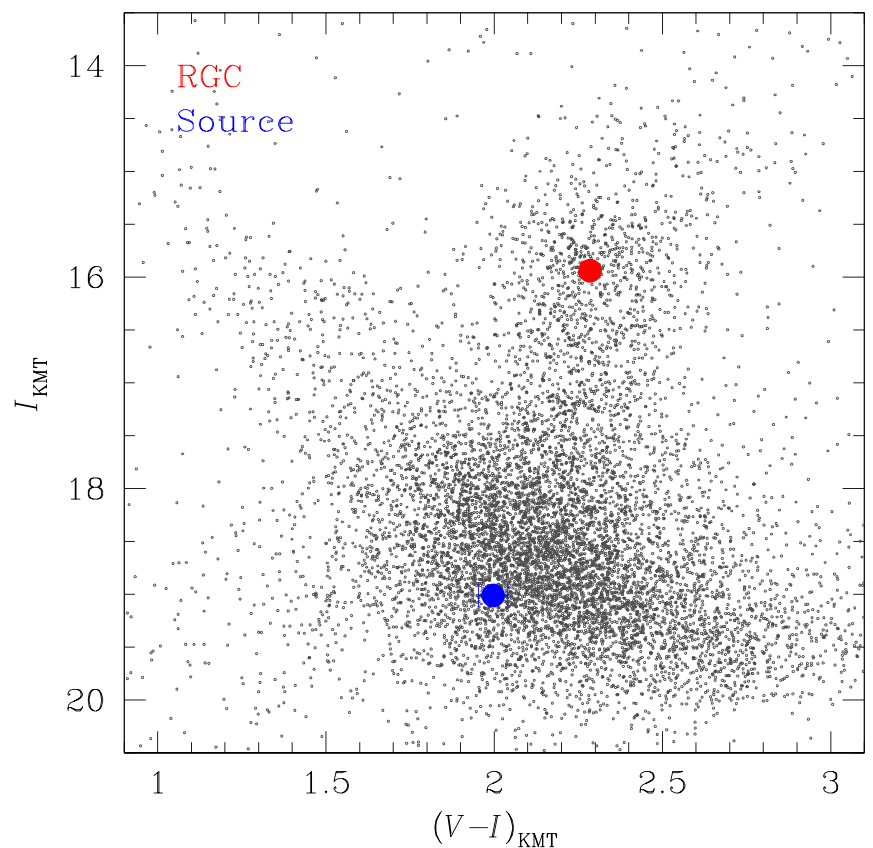

Figure 7. Position of the source star in the instrumental color-magnitude diagram constructed based on the KMTNet data. Also marked is the location of red giant clump (RGC) centroid.

parameters because it is related to the parameters by

$$
t_{\mathrm{E}}=\frac{\sqrt{\kappa M \pi_{\mathrm{rel}}}}{\mu} ; \quad \pi_{\mathrm{rel}}=\mathrm{au}\left(\frac{1}{D_{\mathrm{L}}}-\frac{1}{D_{\mathrm{S}}}\right) .
$$

Here $\mu$ represents the relative lens-source proper motion. We also use the constraint of $\theta_{\mathrm{E}, \mathrm{min}}$.

Implementing a Bayesian analysis requires models describing how lens objects are distributed, i.e., physical distribution, and how they move, i.e., dynamical distribution. One also needs a model mass function of lens objects. We construct the lens mass function based on the Chabrier (2003) mass function for stars combined with the Gould (2000a) mass function for stellar remnants including black holes, neutron stars, and white dwarfs. Lens and source objects are assumed to be distributed following the physical matter distribution model of Han \& Gould (2003), in which the disk has a double-exponential form and the bulge has a triaxial shape. For the dynamical distribution, we adopt Han \& Gould (1995) model, in which the motion of disk objects follows a Gaussian velocity distribution with a mean corresponding to the rotation speed of the disk, and the motion of bulge objects follows a triaxial Gaussian distribution with the velocity components along the individual axes deduced from the bulge shape via the tensor virial theorem. Based on the model distributions, we conduct a Monte Carlo simulation to generate a large number $\left(6 \times 10^{6}\right)$ of Galactic lensing events. Then, the the lens mass and distance distributions are constructed based on the events with timescales located within the range of the measured event timescale. With these distributions, we then estimate the representative values of $M$ and $D_{\mathrm{L}}$ as the median values. The lower and upper limits of the values are estimated as the $16 \%$ and $84 \%$ of the distribution.

In Figure 8, we present the distributions of the lens mass (upper panel) and distance (lower panel) obtained from the Bayesian analysis. In each panel, the blue curve is the distribution based on only the event timescale $t_{\mathrm{E}}$, while the red 

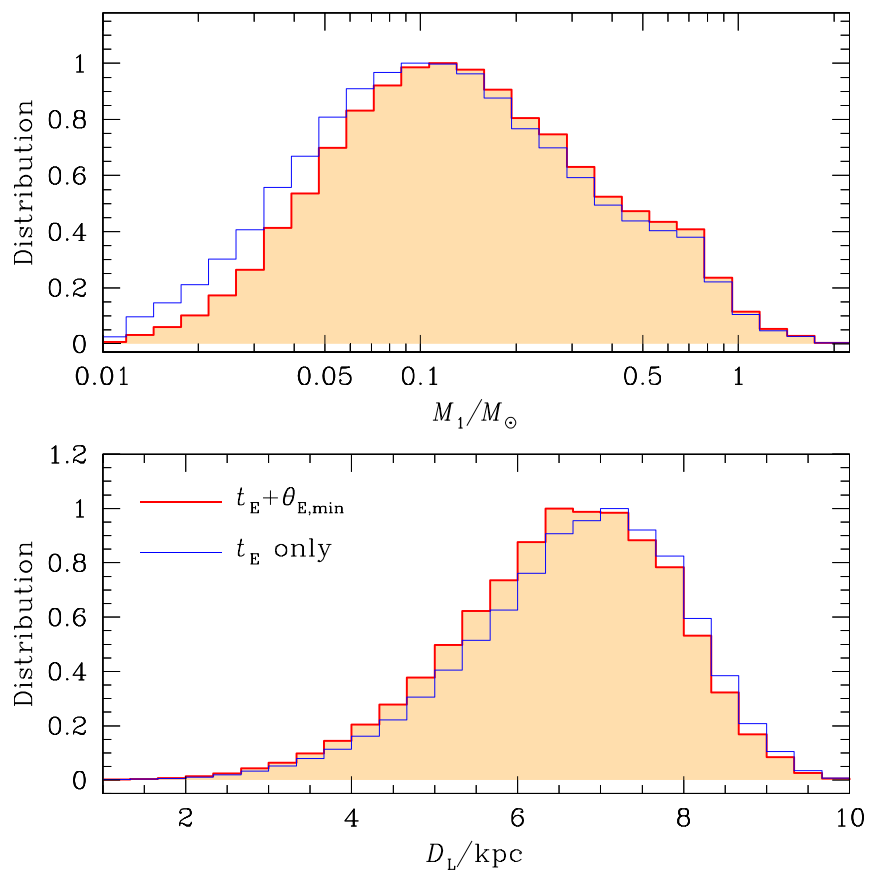

Figure 8. Probability distributions of the mass (upper panel) and distance (lower panel) of the planet host. The blue curve is the distribution based on only the event timescale $t_{\mathrm{E}}$, while the red curve is the distribution with the additional constraint of the lower limit of the angular Einstein radius, $\theta_{\mathrm{E}, \mathrm{min}}$.

curve is the distribution obtained with the additional constraint of $\theta_{\mathrm{E}, \mathrm{min}}$. It is found that the constraint of $\theta_{\mathrm{E}, \min }$ is weak and thus has little effect on the probability distribution. The estimated masses of the lens components are

$$
M_{1}=0.15_{-0.08}^{+0.28} \quad M_{\odot}
$$

and

$$
M_{2}=0.62_{-0.33}^{+1.16} M_{\mathrm{J}}
$$

Therefore, the lens is a planetary system in which a giant planet is orbiting a low-mass $\mathrm{M}$ dwarf. Planetary systems belonging to low-mass hosts are difficult to be detected by other major planet detection methods, e.g., radial velocity or transit methods, due to the faintness of host stars. On the other hand, the microlensing method does not rely on the brightness of the host star, and thus the majority of planetary systems with lowmass stars were found using the microlensing method. See Figure 10 of Han et al. (2018) and Figure 6 of Jung et al. (2018), which show the distribution of planets in the $M_{1}-M_{2}$ plane. The planetary system is estimated to be located at a distance of

$$
D_{\mathrm{L}}=6.8_{-1.4}^{+1.2} \mathrm{kpc} \text {. }
$$

In Table 3, we list the physical lens parameters. We note that the ranges of the lens mass and distances are considerable due to the Bayesian nature of determining the lens parameters combined with weak constraint of extra information, e.g., $\theta_{\mathrm{E}}$ or $\pi_{\mathrm{E}}$. Also presented in the table is the projected separation between the planet and host, which is estimated by $a_{\perp}=s D_{\mathrm{L}} \theta_{\mathrm{E}}$. The projected separation is $a_{\perp} \sim 0.95$ and $\sim 1.05$ au for the inner and outer solutions, respectively. In both cases, the planet is located away from the snow line at $a_{\text {snow }} \simeq 2.7 \mathrm{au}\left(M / M_{\odot}\right) \sim 0.4 \mathrm{au}$.
Table 3

Physical Lens Parameters

\begin{tabular}{lcc}
\hline \hline Parameter & Inner Solution & Outer Solution \\
\hline$M_{1}\left(M_{\odot}\right)$ & $0.15_{-0.08}^{+0.28}$ & $0.15_{-0.08}^{+0.28}$ \\
$M_{2}\left(M_{\mathrm{J}}\right)$ & $0.62_{-0.33}^{+1.16}$ & $0.65_{-0.35}^{+1.21}$ \\
$D_{\mathrm{L}}(\mathrm{kpc})$ & $6.8_{-1.4}^{+1.2}$ & $6.8_{-1.4}^{+1.2}$ \\
$a_{\perp}(\mathrm{au})$ & $0.95_{-0.20}^{+0.17}$ & $1.09_{-0.22}^{+0.19}$ \\
\hline
\end{tabular}

\section{Summary}

We presented the analysis of the planetary lensing event MOA-2016-BLG-319 for which the light curve was characterized by a short-term anomaly near the peak produced by the minor-image perturbation. From modeling of the light curve, we found that there existed two distinct solutions that described the observed light curve almost equally well. The planet-host separations of both solutions were smaller than the Einstein radius, indicating that the degeneracy was different from the previously known "close/wide" degeneracy. From the investigation of the lens-system configurations, it was found that the two solutions resulted from the degeneracy in minor-images perturbations. Because it had been believed that the degeneracy in determining the planet parameters would not be severe for minor-image perturbations, such a degeneracy was unexpected. From the comparison of the lens-system configuration with those of OGLE-2016-BLG-1067 and OGLE-2012-BLG-0950, for which similar degeneracies were reported, we found that the degeneracies for the events were caused by the special source trajectories that passed the star-planet axis at approximately right angles. By conducting a Bayesian analysis, we estimated that the lens was a planetary system in which a giant planet with a mass $M_{\mathrm{p}}=0.62_{-0.33}^{+1.16} M_{\mathrm{J}}\left(0.65_{-0.35}^{+1.21} M_{\mathrm{J}}\right)$ was orbiting a low-mass $\mathrm{M}$ dwarf with a mass $M_{\mathrm{h}}=0.15_{-0.08}^{+0.28} M_{\odot}$, where the planet masses in and out of the parentheses represent the masses for the inner and outer solutions, respectively. The projected host-planet separations were $a_{\perp} \sim 0.95 \mathrm{au}$ and $\sim 1.05$ au for the individual degenerate solutions. The identified degeneracy indicated the need to check similar degeneracies in future analysis of planetary events with minor-image perturbations.

Work by C.H. was supported by the grant (2017R1A4A1015178) of National Research Foundation of Korea. Work by A.G. was supported by US NSF grant AST-1516842. Work by I.G.S. and A.G. was supported by JPL grant 1500811 . This research has made use of the KMTNet system operated by the Korea Astronomy and Space Science Institute (KASI) and the data were obtained at three host sites of CTIO in Chile, SAAO in South Africa, and SSO in Australia. The MOA project was supported by JSPS KAKENHI grant No. JSPS24253004, JSPS26247023, JSPS23340064, JSPS15H00781, and JP16H06287. D.P.B., A.B., and C.R. were supported by NASA through grant NASA80NSSC18K0274. The work by C.R. was supported by an appointment to the NASA Postdoctoral Program at the Goddard Space Flight Center, administered by USRA through a contract with NASA. N.J.R. is a Royal Society of New Zealand Rutherford Discovery Fellow. We acknowledge the high-speed internet service (KREONET) provided by Korea Institute of Science and Technology Information (KISTI). 


\section{Appendix Types of Planetary Perturbations}

When a source star is microlensed, the image of the source splits into two. One image with a higher magnification ("major image") appears outside the Einstein ring and the other image with a lower magnification ("minor image") appears inside the ring. Planetary perturbations occur at the time when the planet is positioned near one of the two microlens images of the primary star and additionally perturbs the nearby image (Mao \& Paczyński 1991; Gould \& Loeb 1992).

Depending on which image is perturbed by the planet, planetary perturbations are classified into two types: "majorimage perturbation" and "minor-image perturbation" (GG1997). The major-image perturbation indicates the case in which the major image is perturbed by the planet. Because the major image is located outside the Einstein ring, major-image perturbations are caused by planets with separations $s>1$. On the other hand, minor-image perturbation indicates the case in which the minor image is perturbed by the planet. The minor image is located inside the Einstein ring and thus minor-image perturbations are caused by planets with separations $s<1$. When a major image is perturbed, the image is further magnified by the planet and thus the lensing light curve always exhibits positive deviations from the light curve produced by the host. In contrast, the minor-image perturbation causes the demagnitification of the image, producing, in most cases, a negative deviation in the light curve.

In the view point on the source plane, planetary lensing signals are produced when a source approaches the caustic produced by the planet. Planets induce one or two sets of "planetary caustics" depending on whether the planetary separation is greater or smaller than the Einstein radius. ${ }^{27}$ For the lens system with a wide planet, there exists a single set of planetary caustics with four cusps. For the system with a close planet, on the other hand, there exist two sets of caustics in which one is located above the planet-host axis and the other is located below the axis and each of the caustics is composed of three cusps. For the detailed properties of planetary caustics, see Han (2006).

\section{ORCID iDs}

Cheongho Han $\odot$ https://orcid.org/0000-0002-2641-9964 Michael D. Albrow (1) https://orcid.org/0000-0003-3316-4012 Sun-Ju Chung (1) https://orcid.org/0000-0001-6285-4528 Kyu-Ha Hwang (i) https://orcid.org/0000-0002-9241-4117 Yoon-Hyun Ryu (i) https://orcid.org/0000-0001-9823-2907 In-Gu Shin iㅏ https://orcid.org/0000-0002-4355-9838 Yossi Shvartzvald (i) https://orcid.org/0000-0003-1525-5041 Jennifer C. Yee (1) https://orcid.org/0000-0001-9481-7123 Richard W. Pogge (1) https://orcid.org/0000-0003-1435-3053 Chun-Hwey Kim (1) https://orcid.org/0000-0001-8591-4562 David P. Bennett (1) https://orcid.org/0000-0001-8043-8413
Akihiko Fukui $\mathbb{1}$ https://orcid.org/0000-0002-4909-5763

Naoki Koshimoto (1) https://orcid.org/0000-0003-2302-9562

Shota Miyazaki (1) https://orcid.org/0000-0001-9818-1513

Nicholas J. Rattenbury (1) https://orcid.org/0000-0001-

5069-319X

Daisuke Suzuki (10 https://orcid.org/0000-0002-5843-9433

\section{References}

Alard, C., \& Lupton, R. H. 1998, ApJ, 503, 325

Albrow, M. D., Horne, K., Bramich, D. M., et al. 2009, MNRAS, 397, 2099 An, J. H. 2005, MNRAS, 356, 1409

Beaulieu, J.-P., Bennett, D. P., \& Fouqué, P. 2006, Natur, 439, 437

Bennett, D. P., \& Rhie, S. H. 1996, ApJ, 472, 660

Bensby, T., Adén, D., Meléndez, J., et al. 2011, A\&A, 533, A134

Bessell, M. S., \& Brett, J. M. 1988, PASP, 100, 1134

Bond, I. A., Abe, F., Dodd, R. J., et al. 2001, MNRAS, 327, 868

Bond, I. A., Bennett, D. P., Sumi, T., et al. 2017, MNRAS, 469, 2434

Bozza, V., Shvartzvald, Y., Udalski, A., et al. 2016, ApJ, 820, 79

Calchi Novati, S., Suzuki, D., Udalski, A., et al. 2018, arXiv:1801.05806

Chabrier, G. 2003, ApJL, 586, L133

Choi, J.-Y., Shin, I.-G., Han, C., et al. 2012, ApJ, 756, 48

Chung, S.-J., Han, C., Park, B.-G., et al. 2005, ApJ, 630, 535

Dominik, M. 1998, A\&A, 329, 361

Dominik, M. 1999, A\&A, 349, 10

Gaudi, B. S. 1998, ApJ, 506, 533

Gaudi, B. S. 2012, ARA\&A, 50, 411

Gaudi, B. S., \& Gould, A. 1997, ApJ, 486, 85

Gould, A. 1992, ApJ, 392, 442

Gould, A. 1994, ApJL, 421, L75

Gould, A. 2000a, ApJ, 535, 928

Gould, A. 2000b, ApJ, 535, 928

Gould, A., \& Horne, K. 2013, ApJL, 779, L28

Gould, A., \& Loeb, A. 1992, ApJ, 396, 104

Griest, K., \& Safizadeh, N. 1998, ApJ, 500, 37

Han, C. 2006, ApJ, 638, 1080

Han, C., \& Gaudi, B. S. 2008, ApJ, 689, 53

Han, C., \& Gould, A. 1995, ApJ, 447, 53

Han, C., \& Gould, A. 2003, ApJ, 592, 172

Han, C., Hirao, Y., Udalski, A., et al. 2018, AJ, 155, 211

Hwang, K.-H., Choi, J.-Y., Bond, I. A., et al. 2013, ApJ, 778, 55

Hwang, K.-H., Kim, H.-W., Kim, D.-J., et al. 2018a, arXiv:1802.10246

Hwang, K.-H., Ryu, Y.-H., Kim, H.-W., et al. 2018b, arXiv:1805.08888

Hwang, K.-H., Udalski, A., Bond, I. A., et al. 2018c, AJ, 155, 259

Hwang, K.-H., Udalski, A., Shvartzvald, Y., et al. 2018d, AJ, 155, 20

Jung, Y. K., Udalski, A., Gould, A., et al. 2018, AJ, 155, 219

Kervella, P., Thévenin, F., Di Folco, E., \& Ségransan, D. 2004, A\&A, 426, 297

Kim, D.-J., Kim, H.-W., Hwang, K.-H., et al. 2018a, AJ, 155, 76

Kim, H.-W., Hwang, K.-H., Kim, D.-J., et al. 2018b, AJ, 155, 186

Kim, S.-L., Lee, C.-U., Park, B.-G., et al. 2016, JKAS, 49, 37

Koshimoto, N., Udalski, A., Beaulieu, J. P., et al. 2017, AJ, 153, 1

Mao, S., \& Paczyński, B. 1991, ApJL, 374, L7

Nataf, D. M., Gould, A., Fouqué, P., et al. 2013, ApJ, 769, 88

Paczyński, B. 1986, ApJ, 304, 1

Park, H., Han, C., Gould, A., et al. 2014, ApJ, 787, 71

Refsdal, S. 1966, MNRAS, 134, 315

Shvartzvald, Y., Yee, J. C., Calchi Novati, S., et al. 2017, ApJL, 840, L3

Skowron, J., Ryu, Y.-H., Hwang, K.-H., et al. 2018, AcA, 68, 43

Sumi, T., Abe, F., Bond, I. A., et al. 2003, ApJ, 591, 20

Yee, J. C., Shvartzvald, Y., Gal-Yam, A., et al. 2012, ApJ, 755, 102

\footnotetext{
${ }^{27}$ We note that planets also induce "central caustics" in the region close to the host of the planet. Due to the location of the caustic, planetary signals produced by central caustics always occur near the peak of lensing events with very high magnifications (Griest \& Safizadeh 1998). For the dependency of the location, size, and shape of the central caustic on the star-planet separation $s$ and the planet/star mass ratio $q$, see Chung et al. (2005).
} 